\title{
Bevacizumab in the management of colorectal cancer: A review
}

\author{
Celina S-P Ang, Nancy E Kemeny \\ Department of Medicine, Gastrointestinal Oncology Service, Memorial Sloan-Kettering Cancer Center, New York, NY 10065.
}

Corresponding Author: Nancy E. Kemeny, MD, 1275 York Avenue, New York, NY 10065.

Tel: 212-639-8068, Fax: 212-794-7168, E-mail: kemenyn@mskcc.org

Received: 2011-08-24

DOI : $10.5430 /$ jst.v1n3p120

\begin{abstract} Background

Since its approval in 2004 for the treatment of metastatic colorectal cancer (mCRC), multiple studies have been conducted with the objective of better defining the use of bevacizumab within and beyond the metastatic setting. This review aims to summarize the data on the clinical applications and nuances of bevacizumab in the management of CRC.

Principal Findings

Bevacizumab can prolong survival in patients with metastatic disease, but is not beneficial in the adjuvant setting. Bevacizumab may be considered as part of peri-operative treatment for carefully selected patients undergoing resection of liver metastases. Bevacizumab added to neoadjuvant chemoradiation for localized rectal cancer demonstrates promising activity and safety, but this is investigational.

\section{Conclusions/Significance}

The decision to use bevacizumab in the management of CRC requires careful consideration of the patient and clinical context. Given the cost as well as toxicities of bevacizumab, it is important for oncologists to know how to use this drug judiciously in order to ensure patient safety and quality of life in addition to improving survival.
\end{abstract}

\section{Key words:}

Bevacizumab; Colorectal Cancer; Chemotherapy; Liver Metastases

\section{NTRODUCTI ON}

In recent years, CRC incidence and mortality have declined in North America and Western Europe as a result of better screening strategies and more effective therapies. ${ }^{1}$ However, there are still 150000 new cases diagnosed each year. ${ }^{2}$

The management of metastatic colorectal cancer (mCRC) has evolved substantially over the past decade with the introduction of new chemotherapy combinations and biologic agents. Bevacizumab (Bv) is a recombinant humanized monoclonal antibody targeting vascular endothelial growth factor-A (VEGF-A). ${ }^{3}$ It was first approved by the FDA for clinical use in the management of mCRC in 2004. Since then, Bv has also been approved for other advanced malignancies including non-squamous non-small cell lung cancer, glioblastoma, renal cell carcinoma and HER-2 negative breast cancer. Investigators are also exploring the potential of $\mathrm{Bv}$ to enhance outcomes in CRC beyond the metastatic setting. The objective of this review is to summarize the data on the clinical applications and nuances of $\mathrm{Bv}$ in the management of CRC. 


\section{METASTATI C DI SEASE: PRI MARY THERAPY}

In 2004, a phase III study comparing irinotecan and bolus weekly 5-fluorouracil/leucovorin (IFL) with or without Bv for the first-line treatment of mCRC revealed, in 813 patients, striking improvements in all study endpoints. ${ }^{4}$ Median overall survival (OS; 20.3 vs. 15.8 months, HR 0.66, $P<0.001$ ), median progression-free survival (PFS; 10.6 vs. 6.2 months, HR 0.54, $P<0.001)$, objective responses (44.8\% vs. $34.8 \%, P=0.004)$ and median response duration (10.4 vs. 7.1 months, HR 0.62, $P=0.001$ ) were all significantly increased with the addition of Bv. This study led to the approval of Bv by the United States Food and Drug Administration (FDA) for use in the first-line treatment of mCRC.

$\mathrm{Bv}$ has also been shown to be active in combination with the more contemporary regimen of infusional 5-fluorouracil (5FU), leucovorin (LV) and irinotecan (FOLFIRI) in the first-line setting. The BICC-C trial initially randomized patients to FOLFIRI, modified bolus 5FU/LV and irinotecan (mIFL) or capecitabine and irinotecan (CapeIRI) with an additional randomization to celecoxib or placebo. ${ }^{5}$ Following FDA approval of Bv, the protocol was amended to compare FOLFIRI + Bv with mIFL + Bv; CapeIRI was not evaluated with Bv because of unacceptable toxicity. The most recent update from this study reported superior OS outcomes with FOLFIRI + Bv compared to to mIFL + Bv (28 vs. 19.2 months, HR 1.79, $P=0.037$ ); however, this was a retrospective analysis. Objective and complete response rates for FOLFIRI + Bv vs. mIFL + Bv were similar at 57.9\% vs. 53.3\% and 5.3\% vs. 5.0\%, respectively. ${ }^{6}$

In another retrospective study, Bv also improved outcomes when combined with oxaliplatin-based chemotherapy. The Three Regimens of Eloxatin Evaluation (TREE) studies followed a design analogous to that of the BICC-C study. TREE-1 reported improved response rates, time to treatment failure (TTF), time to progression (TTP), median and 1year OS with infusional 5FU, LV and oxaliplatin (mFOLFOX6) compared to bolus 5-fluorouracil, leucovorin and oxaliplatin (bFOL) and capecitabine/oxaliplatin (CapeOx). In TREE-2, Bv was added to each of these regimens and the dose of capecitabine in the CapeOx regimen was lowered given a grade 3/4 toxicity rate of 67\% in TREE-1. The addition of Bv improved OS when the TREE-2 and TREE-1 cohorts were compared: 23.7 vs. 18.2 months. The highest overall response rates and median survival were observed with mFOLFOX6 + Bv at $52 \%$ and 26.1 months, respectively. ${ }^{7}$

A prospective trial examining the efficacy of Bv with oxaliplatin regimes was not as successful. On NO16966, a phase III trial of 1401 patients were first randomized to either FOLFOX4 or capecitabine/oxaliplatin (XELOX) then to Bv or placebo. ${ }^{8}$ While progression-free survival (PFS) was significantly greater with the addition of Bv to both regimens (9.4 vs. 8.0 months, HR 0.83, $P=0.0023)$, the improvement in OS did not attain statistical significance (21.3 vs. 19.9 months, HR $0.89, P=0.077$ ). Objective response rates were virtually equivalent between the Bv and placebo groups; $47 \%$ and $49 \%$ by investigator assessment, and $38 \%$ for both groups according to the independent review committee. When the FOLFOX and XELOX groups were evaluated separately, there was no difference in PFS, survival or response rate. Protocol compliance was an issue with this study; the investigators noted that upon the development of oxaliplatin neurotoxicity, many patients discontinued therapy completely instead of continuing the fluoropyrimdine and Bv as recommended in the protocol. As a result, only $29 \%$ of patients on Bv discontinued therapy as a result of disease progression compared to $47 \%$ of patients receiving placebo.

The addition of Bv to first-line FOLFOXIRI (infusional 5FU, LV, oxaliplatin and irinotecan) has been evaluated in phase II study with noteworthy results. ${ }^{9}$ The objective response rate was a reported $77 \%$, including complete responses in $12 \%$ of patients. The remaining $23 \%$ of patients had stable disease. Median PFS and OS were 13.1 and 30.9 months, respectively. In phase II and III studies conducted by the same group of investigators, FOLFOXIRI without Bv has previously been reported to produce an objective response rate of 60-72\% and median PFS and OS times of 9.8-10.8 months and 22.6-28.4 months, respectively. ${ }^{10,11}$

The combination of $\mathrm{Bv}$ with fluoropyrimidines only is also an acceptable first-line option for patients unable to tolerate irinotecan or oxaliplatin. Two randomized phase II studies demonstrated significant improvements in PFS and time to progression, as well as higher response rates and trends to longer median OS with Bv and weekly bolus 5FU/LV compared to 5FU/LV alone. ${ }^{12,13}$ Of note, these studies demonstrated that even patients with poorer risk features such as a median age $\geqslant 65$ years, ECOG $>0$ and serum albumin $\leqslant 3.5 \mathrm{mg} / \mathrm{dL}$ stand to benefit from this regimen. A pooled analysis of these trials with the data from the phase III trial by Hurwitz, et al. ${ }^{4}$ (including the $5 F U / \mathrm{LV}+\mathrm{Bv}$ cohort) revealed statistically significant improvements in median survival (17.4 vs. 14.6 months, HR 0.74, $P=0.008$ ), PFS (8.8 vs. 5.6 months, HR 0.63, $P \leqslant 0.0001$ ), and response rates (34\% vs. 25\%, $P=0.019$ ) with the combination of Bv/5FU/LV in previously untreated patients with mCRC. ${ }^{14}$ Combining Bv with capecitabine, with or without mitomycin C, has also been shown to significantly improve PFS (8.5 vs. 5.7 months, HR $0.63, P<0.001$ ) but not OS or response rates. ${ }^{15}$ 


\section{Is Bevacizumab more Useful with I rinotecan or Oxaliplatin?}

The Bevacizumab Expanded Access Trial (BEAT) was an observational cohort study examining the patterns of use, efficacy and safety of Bv combined with standard first-line chemotherapies for mCRC. ${ }^{16}$ The most commonly used chemotherapy regimens were FOLFOX (29\%), FOLFIRI (26\%), capecitabine/oxaliplatin (18\%) and monotherapy (16\%). Median time to progression, PFS and OS for the entire cohort of 1441 patients were 11.3 , 10.8 and 22.7 months, respectively. Breakdown by chemotherapy regimen showed that outcomes were comparable when Bv was combined with any of these doublet regimens. Median time to progression ranged from 11.2-12 months and PFS ranged from 10.8-11.6 months. Median OS for FOLFOX, FOLFIRI and capecitabine/oxaliplatin was 25.9, 23.7 and 23 months, respectively.

Although the BEAT results must be interpreted in the context of the non-controlled setting of an observational study, the similarities in survival times from the various randomized phase III studies support the notion that Bv combined with oxaliplatin or irinotecan are equivalent in efficacy. However, it has been argued that the degree of benefit gained by combining $\mathrm{Bv}$ with oxaliplatin is less than that with irinotecan. In the Hurwitz, et al. study, median PFS and OS were each improved by approximately 4.5 months, and the objective response rate increased by $10 \%$ with the addition of Bv to IFL. ${ }^{4}$ The BICC-C and TREE studies did not include direct comparisons between Bv-containing and non-Bvcontaining chemotherapy regimens. Thus, the only randomized trial comparing oxaliplatin-based chemotherapy with or without Bv was the NO16966 study. While the improvement in PFS with Bv was statistically significant, the difference was only 1.4 months, and there was no difference in response rates or OS. ${ }^{8}$

There have been no head to head trials comparing FOLFOX + Bv with FOLFIRI + Bv that might shed further light on these differences. At the present time, both regimens are considered acceptable for the first-line therapy of mCRC and are endorsed by the National Comprehensive Cancer Network (NCCN) guidelines. ${ }^{17,18}$

\section{Combining Bevacizumab with Anti-EGFR Therapies}

Cetuximab and panitumumab are monoclonal antibodies targeting the epidermal growth factor receptor (EGFR). Both of these agents are FDA approved for the management of KRAS wild-type mCRC. Based on preclinical data showing that inhibition of EGFR signaling can decrease expression of pro-angiogenic factors ${ }^{19}$ and that acquired resistance to therapy with an anti-EGFR antibody therapies may occur through upregulation of angiogenesis, ${ }^{20}$ several clinical trials were conducted to determine whether combining $\mathrm{Bv}$ with cetuximab or panitumumab would improve outcomes in mCRC.

The first of these was the BOND-2 study which was a randomized phase II trial comparing therapy with cetuximab/Bv/irinotecan vs. cetuximab/Bv in 83 patients with irinotecan-refractory mCRC. ${ }^{21}$ The study demonstrated that combined monoclonal antibody therapy was safe and feasible, although the frequency of grade 3 acneiform skin rash appeared to be higher then previously documented. In an exploratory analysis of efficacy, the three-drug compared to two-drug combination tended to improve response rates (37\% vs. 20\%), time to progression (7.3 vs. 4.9 months) and median survival (14.5 vs. 11.4 months).

Subsequently, two randomized phase III studies were performed evaluating combined anti-EGFR agents with Bv and standard chemotherapy for the first-line treatment of mCRC. The Panitumumab Advanced Colorectal Cancer Evaluation (PACCE) study was a community based phase IIIB trial evaluating the contribution of adding panitumumab to Bv and either oxaliplatin/5FU/LV or irinotecan/5FU/LV containing chemotherapy. ${ }^{22}$ The study enrolled a total of 1053 patients, 823 and 230 of whom were allocated to oxaliplatin and irinotecan-based chemotherapy, respectively, based on the preference of the treating physician. Primary endpoints for the oxaliplatin and irinotecan chemotherapy cohorts were median PFS and safety, respectively. Secondary endpoints were response rates, OS and safety. At the first planned interim analysis, a significantly worse PFS was observed in the panitumumab vs. control arm among patients in the oxaliplatin chemotherapy cohort (8.8 vs. 10.5 months, HR 1.44, $P=0.004$ ). Similarly, median PFS was lower in the panitumumab vs. control arm in the irinotecan cohort (10.1 vs. 11.9 months). A higher frequency of pulmonary emboli as well as grade 3/4 dermatologic toxicities, nausea, vomiting, diarrhea, hypomagnesemia and infections were observed on the panitumumab vs. control arm. As a result of these observations, panitumumab was discontinued. However, updated analyses of the data showed continued trends towards inferior outcomes in the panitumumab containing arms. Median OS was worsened with the addition of panitumumab in the oxaliplatin cohort (19.4 vs. 24.5 months) and was unchanged (20.7 vs. 20.5 months) in the irinotecan cohort. An exploratory analysis of KRAS mutation status did not reveal any consistent patterns, although patients with mutated KRAS appeared to do much worse with combined therapy. 
Similar findings were noted in the CAIRO2 study which evaluated capecitabine/oxaliplatin/ $\mathrm{Bv}$ with or without cetuximab. ${ }^{23}$ Progression-free survival was significantly worse with the addition of cetuximab ( 9.4 vs. 10.7 months, $P=$ $0.01)$. The frequency of grade $3 / 4$ toxicities was also greater with cetuximab $(81.7 \%$ vs. $73.2 \%, P=0.006)$ and was reflected in the significantly lesser degree of improvement in quality of life and global health status compared to the control arm. Median OS, response and disease control rates did not differ between the cetuximab and control arms. Several noteworthy findings from subgroup analyses were also reported: 1) Patients with KRAS mutated tumors had considerably shorter PFS and OS when treated with cetuximab; 2) Women treated with cetuximab experienced a shorter PFS compared to the control arm; 3) The grade of dermatologic toxicity showed a significant positive correlation with PFS in patients treated with cetuximab, although the PFS in patients with grade 3 events was ultimately similar to that of patients on the control arm.

The inferior results observed with dual inhibition of EGFR and VEGF mediated signaling were surprising given the compelling preclinical rationale. Several considerations may account for this. In the PACCE study, the intensity and consistency of drug exposure were diminished in the panitumumab arms, likely as a result of toxicity. ${ }^{14}$ Thus, inadequate treatment might be one explanation for the poorer outcomes. Another explanation might be a negative interaction between cetuximab and $\mathrm{Bv}$. The authors of the CAIRO2 study reported a lower frequency of hypertension, a purported marker of $\mathrm{Bv}$ activity, ${ }^{24}$ on the cetuximab arm which is consistent with this hypothesis. It is likely that combined anti-EGFR and anti-VEGF therapy induces other downstream mechanistic changes that may account for these findings, but have yet to be elucidated. Taken together, dual antibody therapy is more toxic, is associated with shorter survival outcomes and cannot be recommended as a therapeutic strategy in mCRC.

\section{Is there a role for Bevacizumab Maintenance Therapy?}

Previously, the paradigm for the management of mCRC was to continue therapy until disease progression and/or unacceptable toxicity. The OPTIMOX1 study ${ }^{25}$ popularized the concept of "stop-and-go" therapy in which period of "induction" chemotherapy (ie. FOLFOX) is followed by "maintenance" with selected components of the original induction regimen (ie. 5FU/LV). This approach was shown to spare patients from cumulative oxaliplatin neurotoxicity without compromising survival outcomes. Given that $\mathrm{Bv}$ is frequently included in the front-line treatment of mCRC, the benefit of $\mathrm{Bv}$ as maintenance therapy has been a subject of interest.

The MACRO study was a randomized phase III non-inferiority study comparing continuous therapy with $\mathrm{Bv}+$ capecitabine/oxaliplatin (XELOX) vs. 6 cycles of $\mathrm{Bv}+$ XELOX followed by maintenance $\mathrm{Bv}$ monotherapy until disease progression. ${ }^{26}$ The results, which were presented at the 2010 Annual ASCO Meeting, did not demonstrate any statistically significant differences in median PFS, OS and response rates between the arms, although the argument for non-inferiority was questioned. ${ }^{27}$ The frequency of grade $3 / 4$ hand-foot syndrome and neurotoxicity were two and threefold higher, respectively, among patients who received continuous $\mathrm{Bv}+\mathrm{XELOX}$ than in those who received maintenance $\mathrm{Bv}$, resulting in a decreased quality of life. Thus, while the toxicities of continuous Bv + XELOX are not acceptable, there is a concern that maintenance therapy with single agent $\mathrm{Bv}$ may compromise outcomes.

Consideration may be given to combining maintenance chemotherapy with Bv. The phase III Combined Oxaliplatin Neurotoxicity Prevention Trial (CONcePT) compared continuous FOLFOX/Bv with an intermittent schedule of oxaliplatin added and removed every 8 cycles. ${ }^{28}$ Patients randomized to the intermittent oxaliplatin arm received a maintenance regimen of $\mathrm{Bv}$ and infusional 5FU/LV. The study was also originally designed to assess the neuroprotective effect of calcium and magnesium infusions. Although the study was prematurely terminated due to initial concerns of inferior outcomes among patients receiving calcium and magnesium, a significant improvement in time to treatment failure was observed on the intermittent oxaliplatin arm compared with continuous FOLFOX/Bv (5.6 vs. 4.2 months, HR 0.58, $P=0.0025)$. In addition, PFS was also lengthened on the intermittent compared to continuous schedule (12 vs. 7.3 months, HR $0.53, P=0.048$ ) although the analysis was limited by the small number of patients and events. Thus, $\mathrm{Bv}$ can be included as part of a fluoropyrimidine maintenance regimen.

\section{Bevacizumab as Part of Second-Line and Third-line Therapy}

The Eastern Cooperative Oncology Group (ECOG) E3200 study randomized 829 patients with mCRC whose disease had progressed on fluoropyrimidine/irinotecan based chemotherapy to Bv alone, FOLFOX4 alone or FOLFOX4 $+\mathrm{Bv}^{29}$ Compared to FOLFOX4, the combination of FOLFOX4 + Bv produced a statistically significant improvement in median OS (12.9 vs. 10.8 months, HR 0.75, $P=0.0011)$, median PFS ( 7.4 vs. 4.7 months, HR $0.61, P<0.0001)$, and response rates $(22.7 \%$ vs. $8.6 \%, P<0.0001)$. The median $\mathrm{OS}$, PFS and response rate with $\mathrm{Bv}$ monotherapy was 10.2 
months, 2.7 months and 3.3\%, respectively. The median OS reported with $\mathrm{Bv}$ alone is thought to have been influenced by post-progression therapy.

Combining Bv with second-line FOLFIRI for the treatment of oxaliplatin-refractory mCRC also appears to be beneficial. A retrospective, single-institution study reported an objective response rate of $23.5 \%$ as well as median PFS and OS of approximately 8.3 and 21.7 months, respectively. ${ }^{30}$

More recently, the phase II BEVACOLOR trial evaluated the efficacy of Bv with second-line irinotecan or oxaliplatinbased chemotherapy in patients who had progressed after first-line treatment with irinotecan or oxaliplatin-based therapy. ${ }^{31}$ The objective response and disease control rates were $32 \%$ and $87 \%$, respectively. The median PFS and OS were 6.5 and 19.3 months, respectively. Grade 3/4 Bv-related toxicities occurred in $9 \%$ of patients and included hypertension, venous and arterial thromboemboli. There were no treatment-related deaths.

$\mathrm{Bv}$ has also been evaluated in the third-line setting with 5FU/LV in patients who have progressed on both oxaliplatin and irinotecan. ${ }^{32}$ Responses were observed in $4 \%$ of patients, and median PFS and OS were 3.5 and 9 months, respectively. Similar results were noted in a retrospective analysis of patients re-treated with either FOLFOX $+\mathrm{Bv}$ or FOLFIRI + Bv after having progressed on both FOLFOX and FOLFIRI. ${ }^{33}$ Of note, $9.5 \%$ of patients had a partial response and 52.4\% experienced stable disease. The median PFS and OS times were 5.3 and 9.5 months, respectively. While the partial response rate achieved in this study was noteworthy in view of previously documented disease refractoriness to all active chemotherapy agents, retreatment with the same agents is a less likely choice nowadays given the availability of anti-EGFR therapies for KRAS wild-type patients.

\section{Continuing bevacizumab after progression in the first-line setting}

Preclinical studies suggest that the effects of anti-angiogenic agents on tumor vasculature are time dependent and that continuous administration is necessary to maintain a suppressive effect. ${ }^{34,35}$ Though prospective data showing supporting the continuation of $\mathrm{Bv}$ beyond disease progression are not available, retrospective observational studies suggest that the aforementioned hypothesis translates into clinical benefit.The Bevacizumab Regimens: Investigation of Treatment Effects and Safety (BRiTE) was an observational cohort study that documented the outcomes of 1445 patients who progressed on first-line Bv containing therapy and were subsequently treated with: 1) no further therapy (17.5\%); 2) post-progression therapy without $\mathrm{Bv}(36.7 \%)$; 3) post-progression therapy with $\mathrm{Bv}$ (44.4\%). ${ }^{36}$ The study showed that patients who received Bv beyond progression experienced the longest survival (31.6 months) compared to those who did not get $\mathrm{Bv}$ beyond progression (19.9 months) or no further therapy (12.9 months). Post-progression Bv containing therapy remained significantly associated with survival beyond progression (HR 0.49, $P<0.001$ ) on multivariate analysis adjusting for pre and post-treatment, patient and site-specific variables. Despite this suggestion of benefit, the conclusions of the BRiTE study have been strongly challenged. ${ }^{37,38}$ Although the authors did attempt to adjust for patient and disease-related factors that might have independently influenced survival as well as the decision on whether or not to administer $\mathrm{Bv}$ beyond progression, this cannot fully overcome the biases inherent in an observational cohort study design. Such a design also limits our ability to draw conclusions about the long-term safety of Bv. Although hypertension was the only side effect that was reportedly increased in the $\mathrm{Bv}$ beyond progression group, the investigators submitted that patients selected to continue $\mathrm{Bv}$ may also have been less susceptible to developing adverse events in the first place. ${ }^{29}$ Furthermore, the mechanism of action of $\mathrm{Bv}$ in humans on tumor cells and their microenvironment in the context of prior exposure to $\mathrm{Bv}$ is unknown, and so a clear scientific basis for this approach has yet to be identified. ${ }^{31}$ In view of these uncertainties, as well as the high cost of $\mathrm{Bv}$, a prospective clinical trial is needed in order to justify the use of Bv beyond progression.

Another observational cohort study evaluating the role of second-line chemotherapy and Bv is the ARIES study presented at the 2010 Annual ASCO Meeting. ${ }^{39}$ In contrast to the BRiTE study in which all patients had received Bv with first-line chemotherapy, ARIES included both Bv-exposed and Bv-naïve patients on second-line therapy. Compared to Bv-naïve patients, Bv-exposed patients exhibited a trend toward improved median OS (21.7 vs. 17.5 months) although there was no difference in PFS (7.6 vs. 8 months). However, a comparison of the profiles of Bv-naïve vs. Bv-exposed patients revealed that Bv-naïve patients were more likely to be female, Black, $>75$ years of age, and with a history of venous thromboembolism or gastrointestinal hemorrhage. ${ }^{40}$ These findings underscore the selection biases that can confound the results of observational studies.

The NCCN guidelines for the management of colorectal cancer state that Bv may be considered as part of second line therapy among selected patients who are $\mathrm{Bv}$ naïve. However, treatment with $\mathrm{Bv}$ beyond progression for previously exposed patients is not endorsed. ${ }^{15,16}$ 


\section{Using Bevacizumab in the Peri-operative Setting}

Approximately $60 \%$ of patients with mCRC will develop liver metastases at some point during their disease course. ${ }^{41}$ Of these, only about $20 \%$ have surgically resectable disease at presentation. ${ }^{42}$ Given the possibility of long term survival in the range of $40 \%-60 \%$ at 5 -years, ${ }^{43}$ there is a strong rationale for trying to render unresectable disease resectable.

Improvements in systemic chemotherapy have drastically increased response rates from $<20 \%$ with $5 \mathrm{FU}{ }^{34}$ to $60 \%$ with FOLFOXIRI ${ }^{35}$ and exceeding $90 \%$ with combined hepatic arterial infusion and systemic chemotherapy. ${ }^{36}$ Resection rates with contemporary chemotherapy doublets are $10 \%-30 \%,{ }^{34} 36 \%$ with FOLFOXIRI ${ }^{35}$ and $47 \%$ with hepatic arterial infusion and systemic chemotherapy. ${ }^{36}$

Adding Bv to today's most potent chemotherapy combinations appears to be a feasible conversion strategy for unresectable colorectal liver metastases. The safety and activity of this approach has been explored in several retrospective series. In one report, perioperative $\mathrm{Bv}$ plus oxaliplatin and/or irinotecan based chemotherapy produced a response rate of $66 \%$ including complete responses in $5.7 \%$ of patients. ${ }^{44}$ Thirty percent of patients required an intraoperative blood transfusion, and post operative morbidities occurred in $42 \%$ and mainly consisted of infectious complications as well as 2 bile leaks, 2 cases of hepatic decompensation, 1 anastomotic dehiscence, 1 small bowel obstruction managed conservatively. Adverse events directly attributable to $\mathrm{Bv}$ included hypertension in 2 patients, anaphylaxis and severe epistaxis. Four-year survival was 52.5\% after a median follow-up of 2.9 years.

Several retrospective series have compared morbidity rates between patients undergoing liver resection who were treated with perioperative $\mathrm{Bv}$ and those who did not. A matched case-control study from Memorial Sloan-Kettering Cancer Center (MSKCC) reported a post-operative morbidity rate of $40.6 \%$ among patients who received $\mathrm{Bv}$ vs. $37.5 \%$ among the control group $(P=1.0) .{ }^{45}$ The most common complications were infectious in nature, including the two grade 3 abscesses requiring surgical intervention. A review from M.D. Anderson reported no significant differences in operative morbidity between patients who received neoadjuvant chemotherapy with $\mathrm{Bv}$ vs. chemotherapy alone (49\% vs. $43 \%, P=$ 0.22). ${ }^{46}$ Low pre-operative serum albumin and concurrent extrahepatic surgery, but not the use of neoadjuvant $\mathrm{Bv}$, were independently associated with an increased risk of post-operative morbidity.

Despite the apparent absence of differences in morbidity between the $\mathrm{Bv}$ treated and control groups in the abovementioned studies, the safety of perioperative $\mathrm{Bv}$ remains questionable. In the MSKCC series, the frequency of infectious complications was threefold higher in the $\mathrm{Bv}$ vs. control group (18.7 vs. $6.2 \%, P=0.29)$. In the M.D. Anderson series, the percentage of patients who developed grade $3 / 4$ adverse events was doubled in the $\mathrm{Bv}$ vs. control group (19.8\% vs. 9.1\%). ${ }^{44}$ Furthermore, the percentage of infectious complications was $9 \%$ in the Bv group compared to $2 \%$ in the control group $(P=0.26)$. While these differences did not meet the benchmark for statistical significance, they are certainly clinically relevant. ${ }^{47}$ Retrospective studies must be interpreted cautiously as they are known to be subject to biases and are inadequately powered to detect statistical significance.

Impressive results from the first prospective trial of perioperative chemotherapy and Bv have been recently reported. ${ }^{48}$ In this multi-center single-arm phase II study, 46 poor-risk patients with unresectable colorectal liver metastases were treated with pre and postoperative capecitabine/oxaliplatin and Bv. The objective response rate was 78\%. Among those with synchronous and nonsynchronous liver metastases, $67 \%$ and $40 \%$, respectively, were able to undergo resection. Three out of 4 patients who achieved a radiologic complete response remained disease-free after 18-20 months of follow-up; the fourth relapsed with a solitary lesion after 23 months. Progression-free survival and OS at 12 months were $50 \%$ and $86 \%$, respectively. Two patients experienced grade 1-2 wound healing complications. Grade 3 toxicities related to $\mathrm{Bv}$ included 1 gastrointestinal perforation and 2 cases each of hypertension and venous thromboembolism. Two patients experienced grade 4 venous thromboembolic events attributable to Bv. No wound dehiscences, hemorrhage, grade $3 / 4$ perioperative complications or deaths were documented.

The use of perioperative $\mathrm{Bv}$ in an attempt to convert unresectable colorectal liver metastases to resectable disease is a promising approach that can benefit carefully selected patients. Bevacizumab should be held for 6-8 weeks before and after surgery to lessen the risk of bleeding and delayed wound healing. Although total perioperative complications do not appear to be increased in experienced surgical hands, wound healing and infectious complications remain a concern. A prospective randomized trial is needed to better answer the question of whether perioperative $\mathrm{Bv}$ increases operative morbidity or not. 


\section{BEVACI ZUMAB IN THE ADJ UVANT SETTI NG}

Two large, randomized phase III studies evaluating Bv in the adjuvant setting have been completed. The NSABP C-08 study randomized 2672 patients with resected stage II/III CRC to 6 months of mFOLFOX6 with or without 1 year of Bv given every 2 weeks. ${ }^{49}$ The study demonstrated no improvement in 3-year disease-free survival (DFS; 77.4\% vs. 75.5\%) with the addition of $\mathrm{Bv}$ regardless of stage, sex, age or lymph node metastases. The investigators noted a significantly lower hazard ratio for DFS during the first 15 months of the study (HR 0.61, $P<0.001$ ) but not after this point (HR 1.22, $P=0.076$ ), suggesting a time-by-treatment effect. Recurrence rates and distribution, mortality rates, as well as survival at 2 years following recurrence were not significantly different between the control or experimental arms, refuting the notion that $\mathrm{Bv}$ might spawn the development of more aggressive disease.

The AVANT trial randomized 3451 patients with resected stage II/III CRC to one of 3 arms: FOLFOX4 vs. FOLFOX4 $+\mathrm{Bv}$ vs. XELOX + Bv. ${ }^{50}$ Chemotherapy in all arms was administered for 6 months and Bv was given every 3 weeks for a total of 1 year. Overall, the results were remarkably similar to those of the NSABP C-08 trial. Three-year DFS was not improved with the addition of Bv to either FOLFOX4 or XELOX. A transient benefit was seen during the first year of treatment with $\mathrm{Bv}$ which was subsequently lost. Toxicities and the duration of chemotherapy and $\mathrm{Bv}$ did not differ between the control or experimental arms and were therefore not felt to have contributed to the observed findings. The number and distribution of recurrences as well as median survival after recurrence were all similar. However, an interim analysis of post-recurrence survival suggested a slight advantage with FOLFOX4, potentially due to cross-over to Bv. Furthermore, early OS data suggested a detrimental trend in the Bv containing arms compared to FOLFOX4 alone. A recent subgroup analysis of DFS according to gender, age, race, and lymph node status did not reveal any significant differences. ${ }^{51}$

The addition of $\mathrm{Bv}$ to adjuvant systemic chemotherapy has not been shown to improve DFS after resection of early stage CRC and may even worsen outcome. No subgroups have been identified that may potentially benefit from adjuvant Bv. The time-by-treatment effect, while intriguing, highlights our limited understanding of the activity of $\mathrm{Bv}$ (see Section “Why doesn’t Adjuvant Bevacizumab Work?”), but does not support the use of Bv in this setting.

Adjuvant Bv was also studied in combination with hepatic arterial infusion (HAI) of floxuridine (FUDR) and systemic chemotherapy in a randomized phase II trial conducted at MSKCC. ${ }^{52}$ A total of 73 patients with resected colorectal liver metastases were randomized to six, 5-week cycles of HAI FUDR and systemic chemotherapy with or without Bv given on days 15 and 29. Chemotherapy consisted of infusional 5FU/LV and oxaliplatin for treatment-naïve patients; those previously exposed to oxaliplatin were treated with infusional 5FU/LV and irinotecan. The primary endpoint, recurrence-free survival (RFS), was numerically higher at 1 (83\% vs. $71 \%)$ and 4 -years (46\% vs. $37 \%)$ in the control compared to experimental arm, although the difference did not reach statistical significance. Overall survival at 4-years was comparable between the arms ( $85 \%$ vs. $81 \%, P=0.5)$. Patients who received Bv experienced significantly greater biliary toxicity compared to those who did not. This was thought to be the result of HAI FUDR-induced biliary injury compounded by impairment of neo-angiogenesis and healing by the concurrent administration of Bv. Thus, Bv added to adjuvant systemic and HAI chemotherapy increases biliary toxicity without improving RFS or OS following resection of early stage CRC.

\section{Why doesn't Adjuvant Bevacizumab Work?}

The disappointing but intriguing results of NSABP C-08 and AVANT exposed potential differences in the mechanism of action of $\mathrm{Bv}$ between macrometastatic and micrometastatic CRC. These might be related to the effects of $\mathrm{Bv}$ on tumor vasculature which are multi-faceted and poorly understood. One purported mechanism is disruption of angiogenesis and vasoconstriction resulting in hypoxia. ${ }^{28}$ Investigators have speculated that a reduction in tumor perfusion by $\mathrm{Bv}$ may have caused tumor cells to enter a protective dormant state, only to "reawaken" with the removal of Bv. ${ }^{43}$ Another possibility is that vasoconstriction by $\mathrm{Bv}$ may have temporarily decreased the visibility of metastatic lesions on imaging. ${ }^{43}$ On the other hand, Bv may have a "normalizing" effect on tumor vasculature which could theoretically lessen intratumoral interstitial pressure, thereby improving the delivery of chemotherapy. ${ }^{28,53}$ Furthermore, anti-angiogenic agents also appear to have direct modulatory effects on tumor cells as well as the immune system. ${ }^{28}$ Whether there is a dominance of one particular vascular effect over another, as well as the contributions of non-vascular actions in the macro-vs. micrometastatic setting is unknown. Taken together, adjuvant Bv only appears to delay, but not prevent, the onset and/or ability to appreciate recurrent disease and should not be used in this context. 


\section{BEVACI ZUMAB AND RADI ATI ON FOR LOCALI ZED RECTAL CANCER}

The recommended management of localized rectal cancer consists of preoperative concurrent chemoradiation (CRT) with either intravenous 5FU or capecitabine followed by postoperative chemotherapy. The achievement of a pathologic complete response (pCR) after preoperative therapy is an important predictor of improved disease-free and overall survival. ${ }^{54}$ With conventional fluoropyrimidine based CRT, the pCR rate ranges from $15 \%-27 \%{ }^{46}$ The impact of adding $\mathrm{Bv}$ to preoperative therapy for rectal cancer has been reported in several recently published studies.

A two-stage single-arm phase II study of preoperative CRT (45 Gy/25 fractions) with capecitabine ( $825 \mathrm{mg} / \mathrm{m}^{2}$ BID days 1-5 of weeks 1-4 of CRT) and Bv (5 mg/kg days 1, 15 and 29) was recently closed after an interim analysis of the first 8 patients reported excessive toxicities. ${ }^{55}$ Grade 3 intestinal bleeding, diarrhea and grade 3/4 intraabdominal/perianal pain each occurred in $25 \%$ of patients. Two out of the 7 patients who underwent surgery experienced post-operative complications: one had wound dehiscence and the other required additional surgery for an ileus. The pCR rate was $25 \%$.

The combination of capecitabine (825mg/m $\mathrm{m}^{2}$ BID days 1-14 and 22-35), oxaliplatin (50 mg/m² days 1, 8, 22, 29) and Bv (5mg/kg day -14 then days 1, 15 and 29 of CRT) concurrent with radiotherapy (50.4 Gy/28 fractions with a boost) was evaluated in a phase II trial of 42 patients with locally-advanced or low-lying rectal cancer. ${ }^{56}$ This regimen was better tolerated with grade $3 / 4$ diarrhea, pelvic pain and fatigue occurring in $24 \%, 10 \%$ and $10 \%$ of patients, respectively. Ninety-two percent of patients had an R0 resection; 8\% had an R1 resection. The post-operative complication rate was $63 \%$ and was most commonly due to infection (29\%) followed by delayed healing (18\%), anastomotic leak (16\%) and fistulae formation (8\%). Eleven percent required re-operation for abscess or leak drainage. Twenty-three percent of patients had complete regression of their primary tumor, and 18\% had a pCR of both the primary and lymph node disease.

The AVACROSS study was a phase II trial of induction XELOX + Bv followed by concurrent CRT (50.4 Gy/28 fractions with a boost) with capecitabine ( $825 \mathrm{mg} / \mathrm{m}^{2}$ BID throughout CRT) and Bv (5mg/kg day 1 of weeks 1,3 and 5) in locally advanced rectal cancer with high-risk features on pelvic MRI. ${ }^{57}$ Grade $3 / 4$ diarrhea, asthenia, neutropenia and thrombocytopenia occurred in $4 \%-11 \%$ of patients during the induction phase. One patient died from complications of diabetic ketoacidosis and another from sudden death. Grade 3 toxicities during CRT included lymphopenia, tenesmus and hypertriglyceridemia, each occurring in $2.5 \%$ of patients. There were no grade 4 toxicities. Ninety-six patients proceeded to surgery, of which $87 \%$ had completed all planned pre-operative therapy. The pCR rate was $36 \%$ and $98 \%$ had an R0 resection. Of note, 58\% of patients had post-operative complications including infection, anastomotic leaks and stoma complications; $24 \%$ required re-operation.

In summary, the incorporation of $\mathrm{Bv}$ into CRT appears to be feasible and active in selected patients, but post-operative toxicities are high. With the exception of the AVACROSS trial, the pCR rates reported with the addition of Bv appear similar to those achieved without Bv. A randomized phase III trial is required to determine the contribution of Bv to fluoropyrimidinebased CRT for locally advanced rectal cancer. Until then, this approach remains investigational.

\section{BI OMARKERS FOR BEVACIZUMAB I N MCRC}

Currently, there are no validated biomarkers that can help to predict the development of toxicities and/or efficacy in CRC patients treated with bevacizumab. However, several clinical and molecular correlates have been examined and may prove to be of value in the future.

The development of hypertension has been correlated with improved survival in mCRC patients treated with $\mathrm{Bv}$ in several retrospective studies. ${ }^{58-60}$ This supports the hypothesis that Bv may exert its effects through vasoconstriction. Changes in tumor enhancement and microvessel heterogeneity measured by dynamic contrast-enhanced magnetic resonance imaging (DCE-MRI), as well as a reduction in metabolic activity on 18F-FDG-PET have also been observed to correlate with responses to $\mathrm{Bv}$ in patients with mCRC. ${ }^{61,62}$

Low expression levels of carbonic anhydrase IX, a regulator of tissue $\mathrm{pH}$ and a recognized poor prognosticator, have also been associated with improved median PFS and OS. ${ }^{63}$ In contrast, tumor VEGF and thrombospondin-2 levels, microvessel density, KRAS and BRAF mutation status have not been shown to predict outcomes with Bv. ${ }^{57,64,65}$ The AVANT trial has undertaken an extensive biomarker analysis, the results of which are pending. ${ }^{44}$ At the present time, there are no validated clinical or molecular biomarkers that can help to predict the development of toxicities and/or efficacy in patients treated with Bv. 


\section{CONCLUSION}

At the present time, the only established clinical role for $\mathrm{Bv}$ in $\mathrm{CRC}$ is in the first-line management of metastatic disease. $\mathrm{Bv}$ can also be considered as part of second-line therapy in patients naïve to Bv. Carefully selected patients with colorectal liver metastases may benefit from the addition of $\mathrm{Bv}$ to peri-operative chemotherapy. However, $\mathrm{Bv}$ is not useful in the adjuvant setting. The mechanisms of action of Bv are not fully understood, and its activity in CRC appears to be more nuanced than previously recognized. Biomarker development that can help to identify patients with favorable outcomes on $\mathrm{Bv}$ and/or who are at risk for excessive toxicities should be a research priority.

\section{Competing Interests: none}

\section{REFERENCES}

[1] Center MM, Jemal A, Smith RA, Ward E. Worldwide variations in colorectal cancer. CA Cancer J Clin. 2009;59:366-378. http://dx.doi.org/10.3322/caac.20038 PMid:19897840

[2] Jemal A, Siegel R, Xu J, et al. Cancer statistics, 2010. CA Cancer J Clin 2010;60:277-300. http://dx.doi.org/10.3322/caac.20073 PMid:20610543

[3] Ferrara N: Vascular endothelial growth factor: Basic science and clinical progress. Endocr Rev 2004;25:581-611. http://dx.doi.org/10.1210/er.2003-0027 PMid:15294883

[4] Hurwitz H, Fehrenbacher L, Novotny W et al. Bevacizumab plus irinotecan, fluorouracil and leucovorin for metastatic colorectal cancer. N Engl J Med 2004;350:2335-2342. http://dx.doi.org/10.1056/NEJMoa032691 PMid:15175435

[5] Fuchs CS, Marshall J, Mitchell E et al. Randomized, controlled trial of irinotecan plus infusional, bolus, or oral fluoropyrimidines in first-line treatment of metastatic colorectal cancer: results from the BICC-C Study. J Clin Oncol 2007;25:4779-4786. http://dx.doi.org/10.1200/JCO.2007.11.3357 PMid:17947725

[6] Fuchs CS, Marshall J, Barrueco J. Randomized, controlled trial of irinotecan plus infusional, bolus, or oral fluoropyrimidines in first-line treatment of metastatic colorectal cancer: updated results from the BICC-C study. J Clin Oncol 2008;26:689-690. http://dx.doi.org/10.1200/JCO.2007.15.5390 PMid:18235136

[7] Hochster HS, Hart LL, Ramanathan RK et al. Safety and efficacy of oxaliplatin and fluoropyrimidine regimens with or without bevacizumab as first-line treatment of metastatic colorectal cancer: results of the TREE Study. J Clin Oncol 2008;26:3523-3529. http://dx.doi.org/10.1200/JCO.2007.15.4138 PMid:18640933

[8] Saltz L, Clarke S, Diaz-Rubio E et al. Bevacizumab in combination with oxaliplatin-based chemotherapy as first-line therapy in metastatic colorectal cancer: a randomized phase III study. J Clin Oncol 2008;26:2013-2019. http://dx.doi.org/10.1200/JCO.2007.14.9930 PMid:18421054

[9] Masi G, Loupakis F, Salvatore L et al. Bevacizumab with FOLFOXIRI (irinotecan, oxaliplatin, fluorouracil, and folinate) as first-line treatment for metastatic colorectal cancer: a phase 2 trial. Lancet Oncol 2010;11:845-852. http://dx.doi.org/10.1016/S1470-2045(10)70175-3

[10] Masi G, Allegrini G, Cupini S, et al. First line treatment of metastatic colorectal cancer with irinotecan, oxaliplatin and 5-fluorouracil/leucovorin (FOLFOXIRI): Results of phase II study with a simplified biweekly schedule. Ann Oncol 2004;15:1766-1772. http://dx.doi.org/10.1093/annonc/mdh470 PMid:15550581

[11] Falcone A, Ricci S, Brunetti I, et al. Phase III trial of infusional fluorouracil, leucovorin, oxaliplatin, and irinotecan (FOLFOXIRI) compared with infusional fluorouracil, leucovorin, and irinotecan (FOLFIRI) as first-line treatment for metastatic colorectal cancer: the Gruppo Oncologico Nord Ovest. J Clin Oncol 2007;25:1670-1676. http://dx.doi.org/10.1200/JCO.2006.09.0928 PMid:17470860

[12] Kabbinavar FF, Schulz J, McCleod M et al. Addition of bevacizumab to bolus fluorouracil and leucovorin in first-line metastatic colorectal cancer: results of a randomized phase II trial. J Clin Oncol 2005;23:3697-3705. http://dx.doi.org/10.1200/JCO.2005.05.112 PMid:15738537

[13] Kabbinavar FF, Hurwitz HI, Fehrenbacher L et al. A phase II, randomized trial comparing bevacizumab with fluorouracil (FU)/leucovorin (LV) with FU/LV alone in patients with metastatic colorectal cancer. J Clin Oncol 2003;21:60-65. http://dx.doi.org/10.1200/JCO.2003.10.066 PMid:12506171

[14] Kabbinavar FF, Hambleton J, Mass RD et al. Combined Analysis of Efficacy: The Addition of Bevacizumab to Fluorouracil/Leucovorin Improves Survival for Patients With Metastatic Colorectal Cancer. J Clin Oncol 2005;23:3706-3712. http://dx.doi.org/10.1200/JCO.2005.00.232 PMid:15867200 
[15] Tebbutt NC, Wilson K, Gebski VJ et al. Capecitabine, bevacizumab, and mitomycin in first-line treatment of metastatic colorectal cancer: results of the Australasian Gastrointestinal Trials Group Randomized Phase III MAX Study. J Clin Oncol 2010;28:3191-3198. http://dx.doi.org/10.1200/JCO.2009.27.7723 PMid:20516443

[16] Van Cutsem E, Rivera F, Berry S et al. Safety and efficacy of first-line bevacizumab with FOLFOX, XELOX, FOLFIRI and fluoropyrimidines in metastatic colorectal cancer: the BEAT study. Ann Oncol 2009;20:1842-1847. http://dx.doi.org/10.1093/annonc/mdp233 PMid:19406901

[17] NCCN Colon Cancer. http://www.nccn.org/professionals/physician_gls/pdf/colon.pdf. (8 July 2011, date last accessed).

[18] NCCN Rectal Cancer. http://www.nccn.org/professionals/physician_gls/pdf/rectal.pdf. (9 July 2011, date last accessed).

[19] Perrotte P, Matsumoto T, Inoue K et al.Anti-epidermal growth factor receptor antibody C225 inhibits angiogenesis in human transitional cell carcinoma growing orthotopically in nude mice. Clin Cancer Res 1999;5:257-265. PMid:10037173

[20] Viloria-Petit A, Crombet T, Jothy S, et al, Acquired resistance to the antitumor effect of epidermal growth factor receptor-blocking antibodies in vivo: A role for altered tumor angiogenesis. Cancer Res 2001;61:5090-5101. PMid:11431346

[21] Saltz L, Lenz H-J, Kindler HL et al. Randomized phase II trial of cetuximab, bevacizumab, and irinotecan compared with cetuximab and bevacizumab alone in irinotecan-refractory colorectal cancer: the BOND-2 study. J Clin Oncol 2007;25:4557-4561. http://dx.doi.org/10.1200/JCO.2007.12.0949 PMid:17876013

[22] Hecht JR, Mitchell E, Chidiac T et al. A randomized phase IIIB trial of chemotherapy, bevacizumab, and panitumumab compared with chemotherapy and bevacizumab alone for metastatic colorectal cancer. J Clin Oncol 2009;27:672-680. http://dx.doi.org/10.1200/JCO.2008.19.8135 PMid:19114685

[23] Tol J, Koopman M, Cats A et al. Chemotherapy, cetuximab and bevacizumab in metastatic colorectal cancer. J Clin Oncol 2009;360:563-572.

[24] Scartozzi M, Galizia E, Chiorrini S et al. Arterial hypertension correlates with clinical outcome in colorectal cancer patients treated with first-line bevacizumab. Ann Oncol 2009;20:227-230. http://dx.doi.org/10.1093/annonc/mdn637 PMid:18842611

[25] Tournigand C, Cervantes A, Figer A et al. OPTIMOX1: a randomized study of FOLFOX4 or FOLFOX7 with oxaliplatin in a stop-and-go fashion in advanced colorectal cancer--a GERCOR study. J Clin Oncol 2006;24:394-400. http://dx.doi.org/10.1200/JCO.2005.03.0106 PMid:16421419

[26] Tabernero J, Aranda E, Gomez B et al. Phase III study of first-line XELOX plus bevacizumab (BEV) for 6 cycles followed by XELOX plus BEV or single-agent (s/a) BEV as maintenance therapy in patients (pts) with metastatic colorectal cancer (mCRC): The MACRO Trial (Spanish Cooperative Group for the Treatment of Digestive Tumors [TTD]) [abstract]. J Clin Oncol 2010;28:15s.

[27] Venook, AP. Gastrointestinal (Colorectal) Cancer. Oral Abstract Session Discussion. 2010 ASCO Annual Meeting. http://www.asco.org/ASCOv2/MultiMedia/Virtual+Meeting?\&vmview=vm_session_present ations_view\&confID=74\&trackID=13\&sessionID=3415. (9 July 2011, date last accessed)

[28] Grothey A, Hart LL, Rowland KM et al. Intermittent oxaliplatin (oxali) administration and time-to-treatment-failure (TTF) in metastatic colorectal cancer (mCRC): Final results of the phase III CONcePT trial. J Clin Oncol 26: 2008 (May 20 suppl; abstr 4010).

[29] Giantonio BJ, Catalano PJ, Meropol NJ et al. Bevacizumab in combination with oxaliplatin, fluorouracil, and leucovorin (FOLFOX4) for previously treated metastatic colorectal cancer: results from the Eastern Cooperative Oncology Group Study E3200. J Clin Oncol 2007;25:15391544. http://dx.doi.org/10.1200/JCO.2006.09.6305 PMid:17442997

[30] Suenaga M, Matsusaka S, Ueno M et al. Predictors of the efficacy of FOLFIRI plus bevacizumab as second-line treatment in metastatic colorectal cancer patients. Surg Today 2011;41:1067-1074. http://dx.doi.org/10.1007/s00595-010-4432-8 PMid:21773895

[31] Bennouna J, Borg C, Delord JP et al. Bevacizumab Combined with Chemotherapy in the Second-Line Treatment of Metastatic Colorectal Cancer: Results from the Phase II BEVACOLOR Study. Clin Colorectal Cancer 2011 Jul 28.

[32] Chen HX, Mooney M, Boron M et al. Phase II multicenter trial of bevacizumab plus fluorouracil and leucovorin in patients with advanced refractory colorectal cancer: an NCI Treatment Referral Center Trial TRC-0301. J Clin Oncol 2006;24:3354-3360. http://dx.doi.org/10.1200/JCO.2005.05.1573 PMid:16849749

[33] Kang BW, Kim TW, Lee JL et al. Bevacizumab plus FOLFIRI or FOLFOX as third-line or later treatment in patients with metastatic colorectal cancer after failure of 5-fluorouracil, irinotecan, and oxaliplatin: a retrospective analysis. Med Oncol 2009;26:32-37. http://dx.doi.org/10.1007/s12032-008-9077-8 PMid:18498064

[34] Ellis LM, Hicklin DJ. VEGF targeted therapy: mechanisms of anti-tumor activity. Nat Rev Cancer 2008;8:579-591. http://dx.doi.org/10.1038/nrc2403 PMid:18596824

[35] Klement G, Baruchel S, Rak J, et al: Continuous low-dose therapy with vinblastine and VEGF receptor-2 antibody induces sustained tumor regression without overt toxicity. J Clin Invest 2000;105:R15-R24. http://dx.doi.org/10.1172/JCI8829 PMid:10772661 PMCid:517491

[36] Grothey A, Sugrue MM, Purdie DM et al. Bevacizumab beyond first progression is associated with prolonged overall survival in metastatic colorectal cancer: results from a large observational cohort study (BRiTE). J Clin Oncol 2008;26:5326-5334. http://dx.doi.org/10.1200/JCO.2008.16.3212 PMid:18854571 
[37] Kopetz S, Abbruzzese JL. Hidden biases in an observational study of bevacizumab beyond progression. J Clin Oncol 2009;27:1732-1733. http://dx.doi.org/10.1200/JCO.2009.21.2084 PMid:19255299

[38] Ellis LM, Haller D.Bevacizumab beyond progression: does this make sense? J Clin Oncol 2008;26:5313-5315. http://dx.doi.org/10.1200/JCO.2008.17.4540 PMid:18854567

[39] Bekaii-Saab TS, Bendell JC, Cohn JL et al. Bevacizumab (BV) plus chemotherapy (CT) in second-line metastatic colorectal cancer (mCRC): Initial results from ARIES, a second BV observational cohort study (OCS). J Clin Oncol 2010;28:15s.

[40] Bekaii-Saab TS, Bendell JC, Cohn AL et al. Initial results from ARIES, a multi-indication bevacizumab (BV) observational cohort study (OCS): Characteristics of metastatic colorectal cancer (mCRC) patients (pts) receiving BV and chemotherapy (CT) in 2nd line. J Clin Oncol 26: 2008 (May 20 suppl; abstr 15002).

[41] Weiss L, Grundmann E, Torhorst J, et al.Haematogenous metastatic patterns in colonic carcinoma: An analysis of 1541 necropsies. J Pathol 1986; 150:195-203. http://dx.doi.org/10.1002/path.1711500308 PMid:3806280

[42] Bismuth H, Adam R, Levi F, Farabos C, Waechter F, Castaing D, et al. Resection of non-resectable liver metastases from colorectal cancer after neoadjuvant chemotherapy. Ann Surg 1996;224:509-520. discussion 520-522.

[43] Abdalla EK, Adam R, Bilchik AJ, et al. Improving resectability of hepatic colorectal metastases: Expert consensus statement. Ann Surg Oncol 2006;13:1271-1280.

http://dx.doi.org/10.1245/s10434-006-9045-5 PMid:16955381

[44] Chaudhury P, Hassanain M, Bouganim N et al. Perioperative chemotherapy with bevacizumab and liver resection for colorectal liver metastases. HPB 2010;12:37-42. http://dx.doi.org/10.1111/j.1477-2574.2009.00119.x PMid:20495643 PMCid:2814402

[45] D'Angelica M, Kornprat P, Gonen M et al. Lack of evidence for increased operative morbidity after hepatectomy with perioperative use of bevacizumab: a matched case-control study. Ann Surg Oncol 2007;14:759-765. http://dx.doi.org/10.1245/s10434-006-9074-0 PMid:17103075

[46] Kesmodel SB, Ellis LM, Lin E et al. Preoperative bevacizumab does not significantly increase postoperative complication rates in patients undergoing hepatic surgery for colorectal cancer liver metastases. J Clin Oncol 2008;26:5254-5260. http://dx.doi.org/10.1200/JCO.2008.17.7857 PMid:18854565

[47] Kemeny MM. How many patients and how many complications does it take to decide if a drug is safe to use before surgery? J Clin Oncol 2009;27:1917-1918. http://dx.doi.org/10.1200/JCO.2008.21.2811 PMid:19255303

[48] Wong R, Cunningham D, Barbachano Y et al. A multicentre study of capecitabine, oxaliplatin plus bevacizumab as perioperative treatment of patients with poor-risk colorectal liver-only metastases not selected for upfront resection. Ann Oncol 2011 Feb 1.

[49] Allegra CJ, Yothers G, O’Connell MJ et al. Phase III trial assessing bevacizumab in stages II and III carcinoma of the colon: results of the NSABP Protocol C-08. J Clin Oncol 2011;29:11-16. http://dx.doi.org/10.1200/JCO.2010.30.0855 PMid:20940184

[50] De Gramont A, Van Cutsem E, Tabernero J et al. AVANT: Results from a randomized, three-arm multinational phase III study to investigate bevacizumab with either XELOX or FOLFOX4 versus FOLFOX4 alone as adjuvant treatment for colon cancer. J Clin Oncol 29: 2011 (suppl 4; abstr 362).

[51] Andre T, Van Cutsem E, Schmoll H et al. A multinational, randomized phase III study of bevacizumab (Bev) with FOLFOX4 or XELOX versus FOLFOX4 alone as adjuvant treatment for colon cancer (CC): Subgroup analyses from the AVANT trial. J Clin Oncol 29: 2011 (suppl; abstr 3509).

[52] Kemeny NE, Jarnagin WR, Capanu M et al. Randomized phase II trial of adjuvant hepatic arterial infusion and systemic chemotherapy with or without bevacizumab in patients with resected hepatic metastases from colorectal cancer. J Clin Oncol 2011;29:884-889. http://dx.doi.org/10.1200/JCO.2010.32.5977 PMid:21189384

[53] Grothey A, Galanis E. Targeting angiogenesis: progress with anti-VEGF treatment with large molecules. Nat Rev Clin Oncol. 2009;6:507-518. http://dx.doi.org/10.1038/nrclinonc.2009.110 PMid:19636328

[54] Maas M, Nelemans PJ, Valentini V et al.Long-term outcome in patients with a pathological complete response after chemoradiation for rectal cancer: a pooled analysis of individual patient data. Lancet Oncol 2010; 11:835-844. http://dx.doi.org/10.1016/S1470-2045(10)70172-8

[55] Resch G, De Vries A, Ofner D et al. Preoperative treatment with capecitabine, bevacizumab and radiotherapy for primary locally advanced rectal cancer -A two stage phase II clinical trial. Radiother Oncol 2011 Jul 7.

[56] Kennecke H, Berry S, Wong R et al. Pre-operative bevacizumab, capecitabine, oxaliplatin and radiation among patients with locally advanced or low rectal cancer: A phase II trial. Eur J Cancer 2011 Jun 8.

[57] Noque M, Salud A, Vicente P et al. Addition of bevacizumab to XELOX induction therapy plus concomitant capecitabine-based chemoradiotherapy in magnetic resonance imaging-defined poor-prognosis locally advanced rectal cancer: the AVACROSS study. Oncologist 2011;16:614-620. http://dx.doi.org/10.1634/theoncologist.2010-0285 PMid:21467148

[58] Scartozzi M, Galizia E, Chiorrini S et al. Arterial hypertension correlates with clinical outcome in colorectal cancer patients treated with first-line bevacizumab. Ann Oncol 2009;20:227-230. http://dx.doi.org/10.1093/annonc/mdn637 PMid:18842611 
[59] Ryanne Wu R, Lindenberg PA, Slack R et al. Evaluation of hypertension as a biomarker of bevacizumab efficacy. J Gastrointest Cancer 2009;40:101-108.

http://dx.doi.org/10.1007/s12029-009-9104-9 PMid:19921473

[60] Osterlund P, Soveri LM, Isoniemi H et al. Hypertension and Hypertension and overall survival in metastatic colorectal cancer patients treated with bevacizumab-containing chemotherapy. Br J Cancer 2011;104:599-604. http://dx.doi.org/10.1038/bjc.2011.2 PMid:21304526

[61] O’Connor JP, Rose CJ, Jackson A et al.DCE-MRI biomarkers of tumour heterogeneity predict CRC liver metastasis shrinkage following bevacizumab and FOLFOX-6. Br J Cancer 2011;105:139-145. http://dx.doi.org/10.1038/bjc.2011.191 PMid:21673686

[62] Vriens D, de Geus-Oei LF, Heerschap A et al. Vascular and metabolic response to bevacizumab-containing regimens in two patients with colorectal liver metastases measured by dynamic contrast-enhanced MRI and dynamic 18F-FDG-PET. Clin Colorectal Cancer 2011;10:E1-5. PMid:21609927

[63] Hong YS, Cho HJ, Kim SY et al. Carbonic anhydrase 9 is a predictive marker of survival benefit from lower dose of bevacizumab in patients with previously treated metastatic colorectal cancer. BMC Cancer 2009;9:246. http://dx.doi.org/10.1186/1471-2407-9-246 PMid:19619339 PMCid:2719665

[64] Jubb AM, Hurwitz HI, Holmgren EB et al. Impact of vascular endothelial growth factor-A expression, thrombospondin-2 expression, and microvessel density on the treatment effect of bevacizumab in metastatic colorectal cancer. J Clin Oncol 2006;24:217-227. http://dx.doi.org/10.1200/JCO.2005.01.5388 PMid:16365183

[65] Price TJ, Hardingham JE, Lee CK et al.Impact of KRAS and BRAF Gene Mutation Status on Outcomes From the Phase III AGITG MAX Trial of Capecitabine Alone or in Combination With Bevacizumab and Mitomycin in Advanced Colorectal Cancer. J Clin Oncol;29:2675-2682. http://dx.doi.org/10.1200/JCO.2010.34.5520

PMid:21646616 